\title{
Nonlinear Ionization of Molecules by Intense Transform-Limited Gaussian Laser Pulses
}

\author{
Samira Barmaki, Salima Hennani, Marc-André Albert, Stéphane Laulan \\ Laboratoire de Physique Computationnelle et Photonique, Université de Moncton Campus de Shippagan, \\ Shippagan, Canada \\ Email: samira.barmaki@umoncton.ca
}

Received 25 August 2014; revised 21 September 2014; accepted 14 October 2014

Copyright $@ 2014$ by authors and Scientific Research Publishing Inc.

This work is licensed under the Creative Commons Attribution International License (CC BY). http://creativecommons.org/licenses/by/4.0/

(c) (i) Open Access

\begin{abstract}
We present in this paper an investigation of the nonlinear process of above-threshold ionization. The process arises when an atomic or molecular system, exposed to an intense laser pulse, continues to absorb more photons than that needed for the ionization to occur. We trigger this nonlinear process in a simple molecular system by exposing it to an intense transform-limited Gaussian laser pulse of $267-\mathrm{nm}$ wavelength which is the third harmonic of an 800-nm wavelength Tisapphire laser. We explore the characteristics of the process by analyzing the kinetic-energy spectra of the electrons ejected from the molecular system under different laser peak intensities.
\end{abstract}

\section{Keywords}

\section{Nonlinear Ionization Process, Intense Femtosecond Laser Fields, Multiphoton Absorption in Molecular Continuum, Transform-Limited Gaussian Laser Pulse}

\section{Introduction}

The recent progress in laser technology has made possible the generation of laser pulses that are increasingly shorter and intense [1]-[3]. Their characteristics and shape made them ideal tools to probe the ultrafast motion of electrons inside an atomic or a molecular system [4] [5]. Highly nonlinear processes arise when a system is exposed to such pulses. One of the most intriguing nonlinear processes discovered to date is the above-threshold ionization (ATI) process that was first observed by Agostini et al. in 1979, when they were experimentally investigating the irradiation of xenon gas with an intense laser field [6]. The measured kinetic-energy spectrum of the ejected electrons (ATI spectrum) from xenon atoms showed an unusual shape that consists of a series of regularly spaced peaks that are surprisingly separated by exactly the photon frequency of the incident laser field. Since then, more experimental studies have been launched on xenon and other rare gases ( $\mathrm{Kr}, \mathrm{Ar}, \mathrm{Ne}$ ) to explore 
the nonlinear process in greater detail [7]-[9]. These investigations have been accompanied by the launching of a series of theoretical studies that have supported the observations made by the experimentalists [10]-[12]. The findings of all these studies indicate that the ATI process shows quite clearly how an atomic or molecular system, under certain conditions, can successively absorb photons beyond the minimum necessary for the ionization to occur. Different features could appear in the ATI process depending of the characteristics and the shape of the incident laser pulses. In most of the conducted studies, the ATI process was successfully triggered and investigated using transform-limited laser pulses, i.e. unchirped pulses. However, few recent studies have pointed out the efficiency of chirped laser pulses as well in exploring and controlling the process [13]-[17].

In this paper, we numerically investigate the ATI process in the hydrogen molecular ion $\mathrm{H}_{2}^{+}$exposed to an intense transform-limited Gaussian laser pulse of 267-nm wavelength (photons of $4.65 \mathrm{eV}$ ), which corresponds to the third harmonic of an 800-nm wavelength Ti: sapphire laser. Our calculation of the whole electronic structure of the ion is based on a discretization technique using B-spline functions [18] [19]. This technique has proven its efficiency to accurately calculate the density of the continuum electronic states [20]. A realistic and precise representation of the electronic continuum is necessary to adequately describe the absorption of the molecular system of a number of excess photons beyond its ionization threshold which causes the appearance of the ATI process. Our treatment of the laser-ion interaction consists in solving the time-dependent Schrödinger equation (TDSE), describing the motion of the electron in the presence of the laser field. The approach we used to solve the TDSE is based on a spectral method that gives very accurate results and allows easy retrieval of the physical quantities of interest such as the ionization probability and the kinetic-energy spectra of the ejected electrons [15]-[17]. We accurately generate the ATI process under different laser peak intensities. We show how the increase of the laser intensity induces changes in the electronic structure of the ion which affects the shape of the emitted ATI spectra.

Atomic units (a.u.) $\left(\mathrm{e}=\hbar=m_{e}=1\right)$ are used throughout the paper unless otherwise mentioned.

\section{Laser-Ion Interaction Approach}

In our investigation, the laser pulse is assumed to be linearly polarized along the $z$ axis, with its vector potential given by:

$$
\boldsymbol{A}(t)=A_{0} f(t) \sin \left(\omega_{0} t\right) \boldsymbol{e}_{z}
$$

$A_{0}$ is the peak amplitude, which is related to the laser central frequency $\omega_{0}$ and the peak intensity $I$ by:

$$
A_{0}=\frac{1}{\omega_{0}} \sqrt{\frac{I}{I_{\text {a.u. }}}}
$$

$I_{\text {a.u. }}=3.51 \times 10^{16} \mathrm{~W} \cdot \mathrm{cm}^{-2}$ is the atomic unit of intensity.

We consider the laser pulse having a Gaussian time envelope $f(t)$ identical to that given in [21] [22]:

$$
f(t)=\exp \left[-2 \ln 2 \frac{t^{2}}{\tau_{0}^{2}}\right]
$$

$\tau_{0}$ is the FWHM duration of the transform-limited pulse of frequency bandwidth:

$$
\Delta \omega=\frac{4 \ln 2}{\tau_{0}}
$$

The kinetic-energy spectrum of the ejected electrons, i.e. the ATI spectrum, is calculated by using the following expression:

$$
\frac{\mathrm{d} P}{\mathrm{~d} E}=\rho(E) \sum_{E>0}\left|\left\langle\Psi\left(\boldsymbol{r}, R, t_{\text {final }}\right) \mid \psi(\boldsymbol{r})\right\rangle\right|^{2}
$$

where $\rho(E)$ is the density of continuum states [20] and $\psi(\boldsymbol{r})$ the electronic stationary wave function associated to the electronic state of energy $E . \Psi(r, R, t)$ is the time-dependent electronic wave function describing the motion of the electron in the laser field. $\boldsymbol{r}$ is the electron radial vector and $R$ the internuclear distance that we 
have fixed to 2 a.u. (the equilibrium distance).

\section{Results and Discussion}

To trigger the nonlinear ATI process, we have exposed the hydrogen molecular ion to a transform-limited Gaussian laser pulse of photon frequency $\omega_{0}=0.171$ a.u. $\left(4.65 \mathrm{eV}\right.$ ), FWHM duration $\tau_{0}=120$ a.u. (2.9 fsec) and peak intensity $I=1 \times 10^{14} \mathrm{~W} \cdot \mathrm{cm}^{-2}$. Figure 1 (a) depicts the variation of the vector potential $\boldsymbol{A}(t)$ in time, see Equation (1), and the spectral profile of the laser pulse. In this case, the molecular system of ionization potential $I_{p}=1.1026$ a.u. $(30 \mathrm{eV})$ has to absorb 7 photons for the ionization to occur.

We show, in Figure 1(b), the ATI spectrum obtained at the end of the laser pulse interaction with the ion. The ATI spectrum exhibits a series of successive peaks localized around some specific electronic energies. All these peaks are the consequence of the excessive absorption of the ion of additional photons beyond the minimum of 7 needed for the ionization to occur. Here, the molecular ion absorbs more than 9 additional photons above its ionization threshold.

We also have exposed the molecular ion to a laser pulse of intensity $I=2 \times 10^{14} \mathrm{~W} \cdot \mathrm{cm}^{-2}$ that we have in-

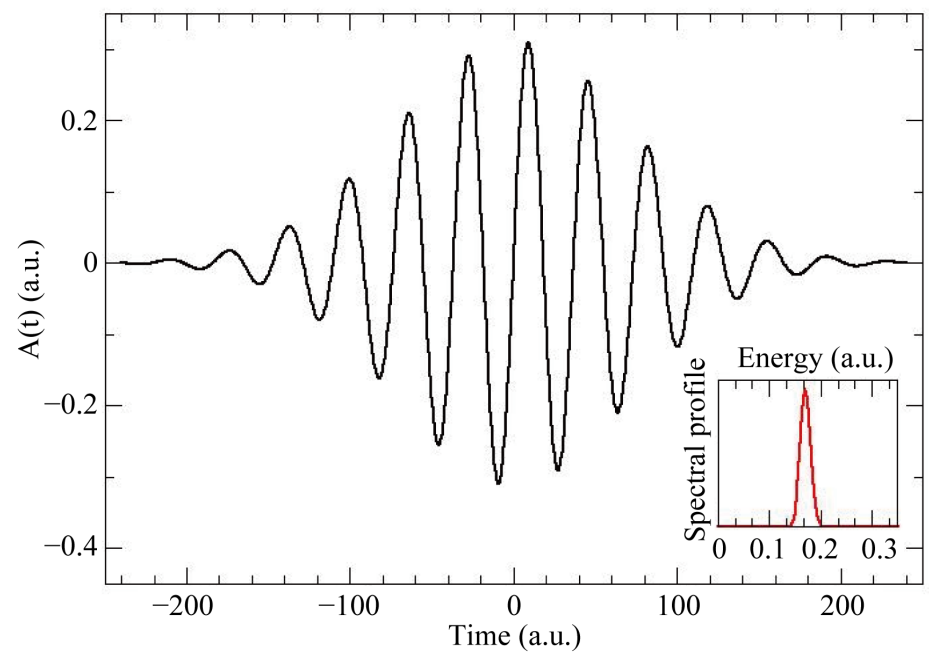

(a)

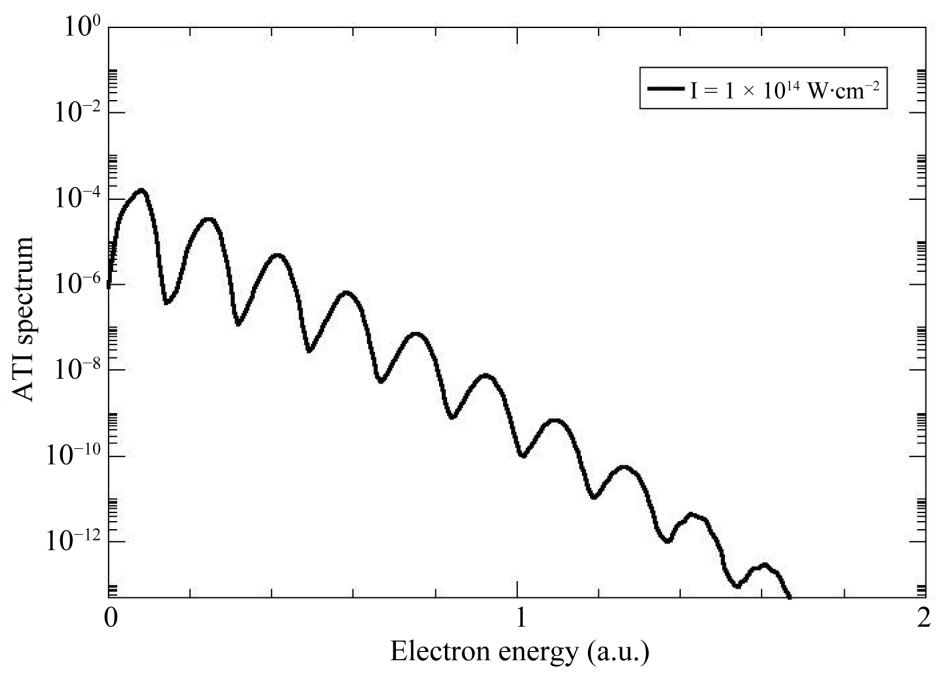

(b)

Figure 1. (a) Variation of the laser pulse vector potential $A(t)$ in time. The laser pulse has a central frequency $\omega_{0}=0.171$ a.u. $(4.65 \mathrm{eV})$, peak intensity $I=1 \times 10^{14} \mathrm{~W} \cdot \mathrm{cm}^{-2}$, and FWHM duration $\tau_{0}=120$ a.u. (2.9 fsec); (b) ATI spectrum generated by the pulse. 
creased later to $I=4 \times 10^{14} \mathrm{~W} \cdot \mathrm{cm}^{-2}$. In both experiments, we have used the same laser frequency and FWHM duration than before. We show, in Figure 2, the obtained ATI spectrum at the end of each experiment. We notice from the figure that the more we increase the intensity, the more the ATI spectrum gains in intensity and the peaks shift to the left. The shift is due to the Stark effect which consists, see Figure 3, in the displacement that electronic states undergo in the presence of an electric field. The ion under the laser pulse of intensity $I$ and photon frequency $\omega_{0}$ sees its ionization threshold displaced by the following amount of energy:

$$
U_{p}=\frac{I}{4 \omega_{0}^{2} I_{\text {a.u. }}}
$$

which is the average energy that the electron of the molecular ion acquires when it is submitted to the electric field.

Figure 3 indicates the changes that occur in the electronic structure of the ion depending of the magnitude of the laser intensity. When the pulse is of $I=1 \times 10^{14} \mathrm{~W} \cdot \mathrm{cm}^{-2}$ and $I=2 \times 10^{14} \mathrm{~W} \cdot \mathrm{cm}^{-2}$, the ionization potential

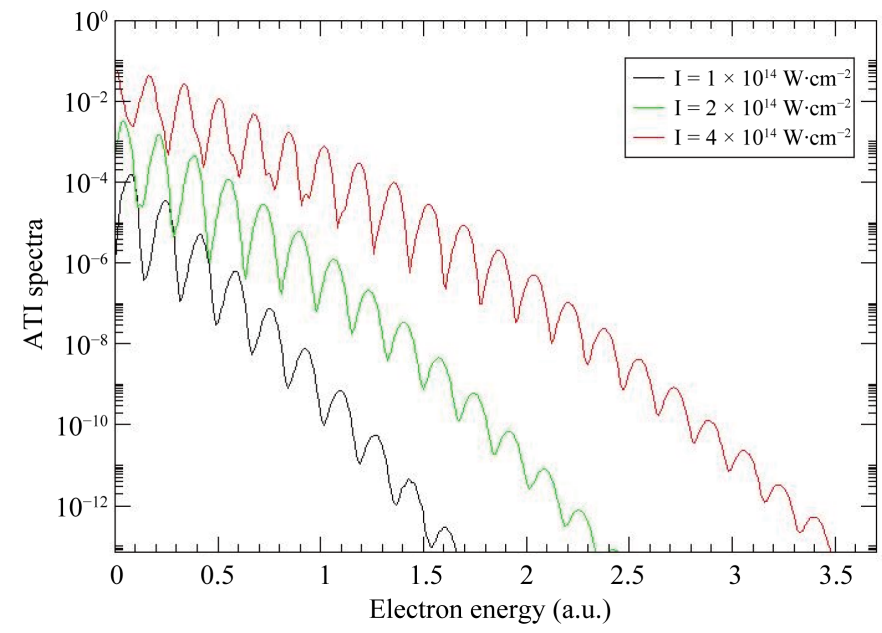

Figure 2. ATI spectra emitted from the molecular ion exposed to a laser pulse of photon frequency $\omega_{0}=0.171$ a.u ( $\left.4.65 \mathrm{eV}\right)$, FWHM duration $\tau_{0}=120$ a.u. (2.9 fsec) and for three different intensities: $I=$ $1 \times 10^{14} \mathrm{~W} \cdot \mathrm{cm}^{-2}, I=2 \times 10^{14} \mathrm{~W} \cdot \mathrm{cm}^{-2}$ and $I=4 \times 10^{14} \mathrm{~W} \cdot \mathrm{cm}^{-2}$.

(c)

(b)

(a)

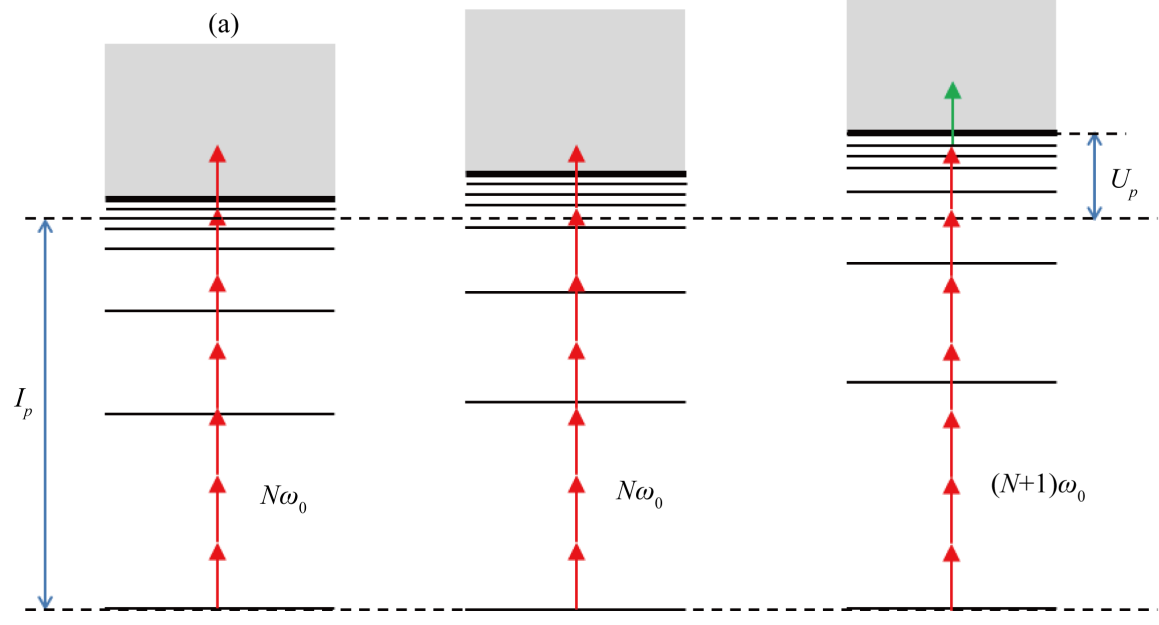

Figure 3. Electronic structure of the ion when submitted to a pulse of intensity (a) $I=1 \times$ $10^{14} \mathrm{~W} \cdot \mathrm{cm}^{-2}$; (b) $I=2 \times 10^{14} \mathrm{~W} \cdot \mathrm{cm}^{-2}$; and (c) $I=4 \times 10^{14} \mathrm{~W} \cdot \mathrm{cm}^{-2}$. 
$I_{p}$ of the ion get increased by $U_{p}=0.024$ a.u. and 0.048 a.u., respectively. In both cases, the absorption of 7 photons is largely enough to ionize the ion. However, when the intensity is increased to $I=4 \times 10^{14} \mathrm{~W} \cdot \mathrm{cm}^{-2}$, the $U_{p}$ increases to 0.097 a.u., here the absorption of 7 photons is no longer enough for the ionization to occur. The ion has to absorb an $8^{\text {th }}$ additional photon to eject the electron. Following this step, in each of the three above studied cases, the nonlinear ATI process is triggered as the ion continues to absorb a number of additional photons beyond its ionization threshold of $I_{p}+U_{p}$ as it is schematically indicated in Figure 4. The energy of the ejected electron by the laser pulse can be then predicted by the simple following formula:

$$
E^{S}=\left(N^{\prime}+S\right) \omega_{0}-\left(I_{p}+U_{p}\right)
$$

where $N^{\prime}$ is the minimum number of photons necessary for the ionization to take place, and $S$ the number of additional absorbed photon by the molecular ion.

Our numerical treatment of the laser pulse interaction with the molecular ion generates precise ATI spectra. The results, presented in Figure 2, accurately recreate the multiphoton absorption in the molecular continuum for three different laser peak intensities. They precisely indicate the kinetic energies $E^{S}$ acquired by electrons when leaving the ion. For example, when the laser peak intensity is set to $I=1 \times 10^{14} \mathrm{~W} \cdot \mathrm{cm}^{-2}$, the first peak of the ATI spectrum results from the absorption of a number of 7 photons and is localized around the kinetic energy $E^{S=0}=0.07$ a.u. The second peak results from the absorption in the molecular continuum of an additional $8^{\text {th }}$ photon and is localized around $E^{S=1}=0.24$ a.u. $=E^{S=0}+\omega_{0}$, and so on for each subsequent peak. The interval of energy $\Delta E=E^{S+1}-E^{S}$ between each two successive ATI peaks is exactly equal to the photon frequency $\omega_{0}$. The results of $E^{S=0}, E^{S=1}, E^{S=2}, \cdots$ we obtained are in agreement with those predicted by Equation (7). The noticeable shift shown in Figure 2 of the ATI peaks obtained for $I=2 \times 10^{14} \mathrm{~W} \cdot \mathrm{cm}^{-2}$ and $I=4 \times 10^{14} \mathrm{~W} \cdot \mathrm{cm}^{-2}$ compared to when $I=1 \times 10^{14} \mathrm{~W} \cdot \mathrm{cm}^{-2}$ is understandable. As seen in Figure 3, the increase of the laser peak intensity significantly displaces the ionization threshold, which shifts the generated ATI peaks towards lower electronic energies $E^{S}$.

\section{Conclusions}

We have investigated the nonlinear ATI process in the hydrogen molecular ion under a laser pulse of 267-nm wavelength. We have generated the ATI spectra under different laser peak intensities with a high degree of accuracy. The obtained spectra are characterized by a series of successive peaks separated exactly by the photon frequency of the incident laser pulse. We have explored the sensitivity of the process to the laser intensity. The more the intensity is increased, the more important the displacement of the ion electronic states becomes which affects the energy of the ejected electrons and then the shape of the ATI spectra.

Our study could be applicable to other simple molecular systems. The case of nonsymmetric molecules will

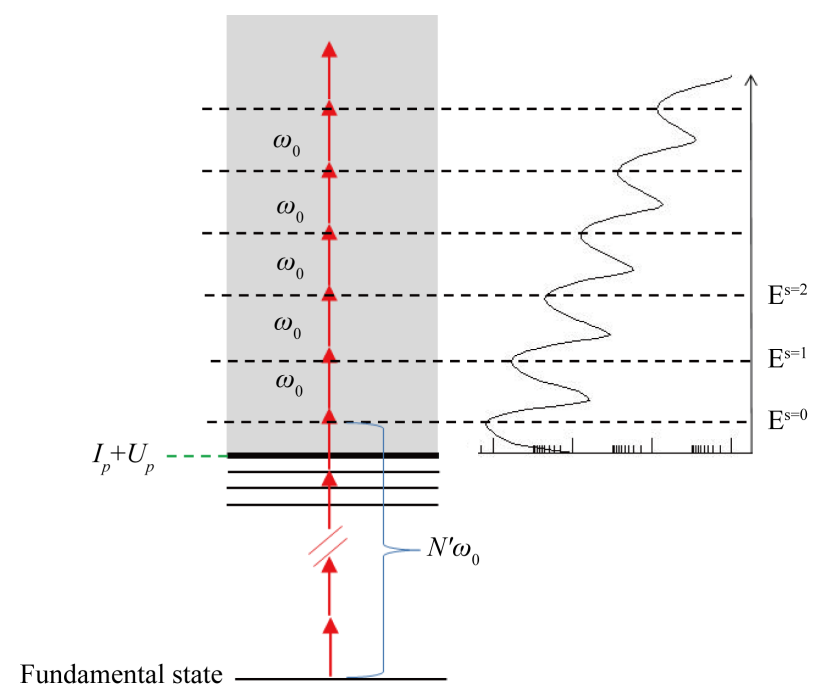

Figure 4. Schematic description of the nonlinear ATI process. 
be interesting as we expect the ATI spectra emitted from these systems to be sensitive to the carrier-envelope phase of a short laser pulse. The appropriate adjustment of this laser parameter will be the key to control the nonlinear behavior of such systems under short and intense laser fields.

\section{Acknowledgements}

The present research was supported by the National Sciences and Engineering Research Council of Canada (NSERC) and by the New-Brunswick Innovation Foundation (NBIF). Allocation of CPU time and assistance with the computer facilities from the Atlantic Computational Excellence Network (ACEnet, St-John's, NL, Canada) and from the "Réseau Québecois de Calcul de Haute Performance" (RQCHP, Sherbrooke, QC, Canada) are acknowledged.

\section{References}

[1] Diels, J.C. and Rudolph, W. (1996) Ultrashort Laser Pulse Phenomenon: Fundamentals, Techniques and Applications on Femtosecond Time Scale. Academic Press, New York.

[2] Agostini, P. and DiMauro, L.F. (2004) The Physics of Attosecond Light Pulses. Reports on Progress in Physics, 67, 813-855. http://dx.doi.org/10.1088/0034-4885/67/6/R01

[3] Yamanouchi, K. and Chin, S.L. (2007) Progress in Ultrafast Intense Laser Science III. Springler-Verlag, Berlin.

[4] Krausz, F. and Ivanov, M. (2009) Attosecond Physics. Review of Modern Physics, 81, 163. http://dx.doi.org/10.1103/RevModPhys.81.163

[5] Barmaki, S., Guessaf, K. and Laulan, S. (2011) Imaging of Ultrafast Electron Motion in Molecules. Canadian Journal of Physics, 89, 703-707. http://dx.doi.org/10.1139/p11-039

[6] Agostini, P., Fabre, F., Mainfray, G., Petite, G. and Rahman, N.K. (1979) Free-Free Transitions Following Six-Photon Ionization of Xenon Atoms. Physical Review Letters, 42, 1127. http://dx.doi.org/10.1103/PhysRevLett.42.1127

[7] Freeman, R.R., Bucksbaum, P.H., Milchberg, H., Darack, S., Schumacher, D. and Geusic, M.E. (1987) AboveThreshold Ionization with Subpicosecond Laser Pulses. Physical Review Letters, 59, 1092-1095. http://dx.doi.org/10.1103/PhysRevLett.59.1092

[8] Schafer, K.J., Yang, B.R., DiMauro, L.F. and Kulander, K.C. (1993) Abovethreshold Ionization beyond the High Harmonic Cutoff. Physical Review Letters, 70, 1599. http://dx.doi.org/10.1103/PhysRevLett.70.1599

[9] Paulus, G.G., Nicklich, W., Zacher, F., Lambropoulos, P. and Walther, H. (1996) High-Order Above-Threshold Ionization of the Atomic Hydrogen Using Intense, Ultrashort Laser Pulses. Journal of Physics B: Atomic, Molecular and Optical Physics, 29, L249-L256.

[10] Javanainen, J., Eberly, J.H. and Su, Q.C. (1988) Numerical Simulations of Multiphoton Ionization and Above-Threshold Electron Spectra. Physical Review A, 38, 3430. http://dx.doi.org/10.1103/PhysRevA.38.3430

[11] Cormier, E., Garzella, D., Breger, P., Agostini, P., Chériaux, G. and Leblanc, C. (2001) Above-Threshold Ionization Contrast and Channel Closure in Argon. Journal of Physics B: Atomic, Molecular and Optical Physics, 34, L9-L17.

[12] Becker, W., Grasbon, F., Kopold, R., Milosevic, D.B., Paulus, G.G. and Walther, H. (2002) Above-Threshold Ionization: From Classical Features to Quantum Effects. Advances in Atomic Molecular and Optical Physics, 48, 35-98. http://dx.doi.org/10.1016/S1049-250X(02)80006-4

[13] Xiang, Y., Niu, Y. and Gong, S. (2009) Above-Threshold Ionization by Few-Cycle Nonlinear Chirped Pulses. Physical Review A, 80, Article ID: 023423. http://dx.doi.org/10.1103/PhysRevA.80.023423

[14] Nakajima, T. (2007) Above-Threshold Ionization by Chirped Laser Pulses. Physical Review A, 75, Article ID: 053409. http://dx.doi.org/10.1103/PhysRevA.75.053409

[15] Laulan, S., Ba, H.S. and Barmaki, S. (2014) Excitation and Ionization of the Hydrogen Atom by Intense Femtosecond Chirped Laser Pulses. Canadian Journal of Physics, 92, 194-199. http://dx.doi.org/10.1139/cjp-2013-0389

[16] Laulan, S., Haché, J., Ba, H.S. and Barmaki, S. (2013) Ionization Process of Atoms by Intense Femtosecond Chirped Laser Pulses. Journal of Modern Physics, 4, 20-26. http://dx.doi.org/10.4236/jmp.2013.410A1002

[17] Barmaki, S., Ba, H.S. and Laulan, S. (2013) Control of the Atomic Ionization with Short and Intense Chirped Laser Pulses. Journal of Modern Physics, 4, 27-31. http://dx.doi.org/10.4236/jmp.2013.410A1003

[18] de Boor, C. (1978) A Practical Guide to Splines. Springer-Verlag, New York.

[19] Bachau, H., Cormier, E., Decleva, P., Hansen, J.E. and Martin, F. (2001) Applications of B-Splines in Atomic and Molecular Physics. Reports on Progress in Physics, 64, 1815-1943. http://dx.doi.org/10.1088/0034-4885/64/12/205

[20] Barmaki, S., Laulan, S., Bachau, H. and Ghalim, M. (2003) The Ionization of One-Electron Diatomic Molecules in 
Strong and Short Laser Fields. Journal of Physics B: Atomic, Molecular and Optical Physics, 36, 817-824. http://dx.doi.org/10.1088/0953-4075/36/5/303

[21] Lee, T.G., Pindzola, M.S. and Robicheaux, F. (2009) Energy and Angular Differential Probabilities for Photoionization of He Using Chirped Attosecond Soft X-Ray Pulses. Physical Review A, 79, Article ID: 053420. http://dx.doi.org/10.1103/PhysRevA.79.053420

[22] Saleh, B.E.A. and Teich, M.C. (2007) Fundamentals of Photonics. John Wiley and Sons Inc., Hoboken. 
Scientific Research Publishing (SCIRP) is one of the largest Open Access journal publishers. It is currently publishing more than 200 open access, online, peer-reviewed journals covering a wide range of academic disciplines. SCIRP serves the worldwide academic communities and contributes to the progress and application of science with its publication.

Other selected journals from SCIRP are listed as below. Submit your manuscript to us via either submit@scirp.org or Online Submission Portal.
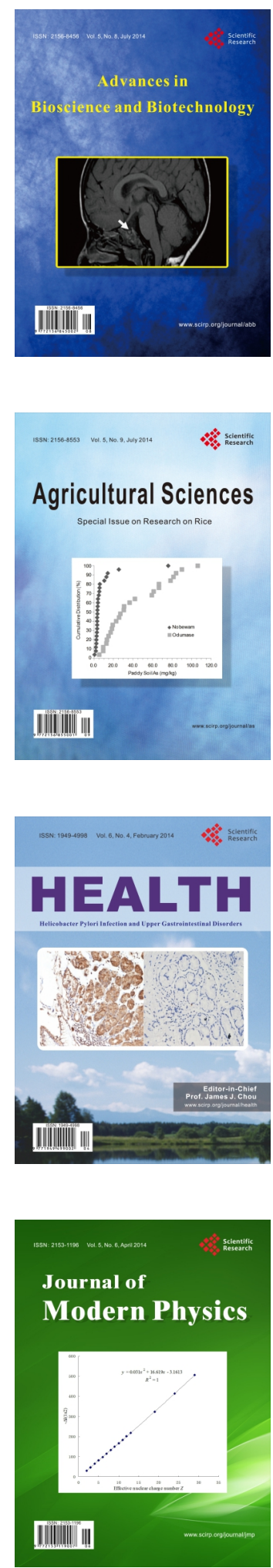
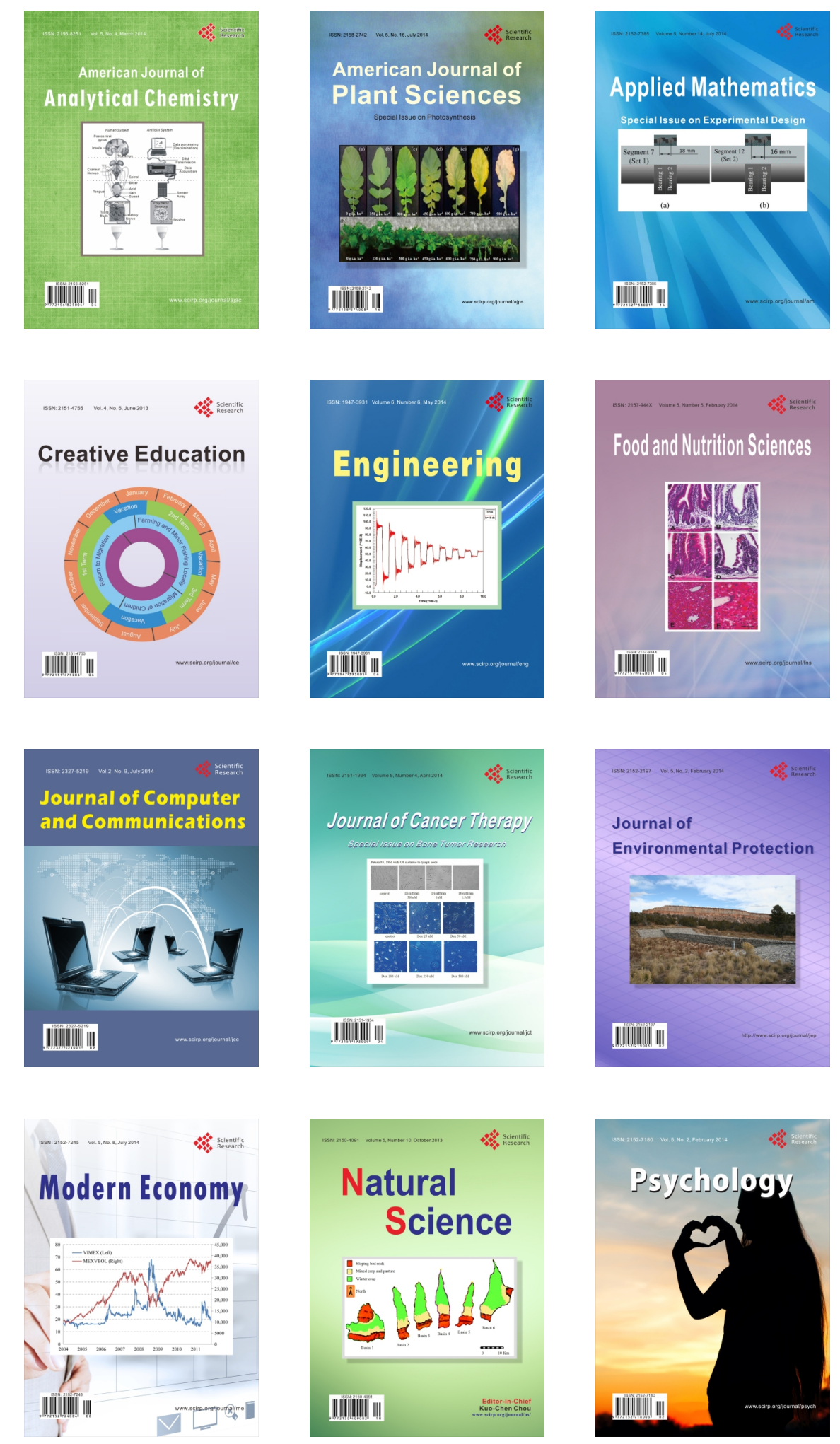